\title{
Comparison of Prasugrel and Ticagrelor for Patients with Acute Coronary Syndrome: A Systematic Review and Meta-Analysis
}

\author{
Lucas Chun Wah Fong ${ }^{a}$ Nicholas Ho Cheung Lee ${ }^{a} \quad$ Andrew T. Yan ${ }^{b}$ \\ Ming-Yen $\mathrm{Ng}^{\mathrm{c}}$
}

aLi Ka Shing Faculty of Medicine, The University of Hong Kong, Hong Kong, China; ${ }^{\mathrm{b}}$ Department of Medicine, University of Toronto Division of Cardiology, St. Michael's Hospital, Toronto, ON, Canada; 'Department of Diagnostic Radiology, Li Ka Shing Faculty of Medicine, The University of Hong Kong, Hong Kong, China

\section{Keywords}

Ticagrelor · Prasugrel · Antiplatelet therapy · Percutaneous coronary intervention - Meta-analysis

\begin{abstract}
Introduction: There have been inconsistent data on the direct comparison of prasugrel and ticagrelor. This meta-analysis was conducted to summarize the current available evidence. Methods: We performed a meta-analysis (PROSPERO-registered CRD42020166810) of randomized trials up to February 2020 that compared prasugrel and ticagrelor in acute coronary syndrome with respect to the composite endpoint of myocardial infarction (MI), stroke, or cardiovascular death and secondary endpoints including MI, stroke, cardiovascular death, major bleeding (Bleeding Academic Research Consortium (BARC) type 2 or above), stent thrombosis, all-cause death, and other safety outcomes. Results: Of the 11 eligible RCTs with 6,098 patients randomized to prasugrel $(n=3,050)$ or ticagrelor ( $n=3,048), 180$ and 207 had the composite endpoint events in the prasugrel arm and the ticagrelor arm, respectively, over a weighted mean follow-up period of $11 \pm 2$ months. Compared with prasugrel, the ticagrelor group had similar risk in the primary compos-
\end{abstract}

karger@karger.com www.karger.com/crd

Karger $\frac{1}{6}$
C 2021 The Author(s).

Published by S. Karger AG, Basel

This article is licensed under the Creative Commons Attribution 4.0 International License (CC BY) (http://www.karger.com/Services/ OpenAccessLicense). Usage, derivative works and distribution are permitted provided that proper credit is given to the author and the original publisher. ite endpoint (risk ratio $[\mathrm{RR}]=1.17 ; 95 \% \mathrm{Cl}=0.96-1.42$; $\left.p=0.12, I^{2}=0 \%\right)$. Compared to prasugrel, there was no significant difference associated with the ticagrelor groups with respect to stroke $(\mathrm{RR}=1.05 ; 95 \% \mathrm{Cl}=0.66-1.67 ; p=0.84$, $\left.R^{2}=0 \%\right)$, cardiovascular death $(\mathrm{RR}=1.01 ; 95 \% \mathrm{Cl}=0.75-1.36$; $\left.p=0.95, P^{2}=0 \%\right)$, BARC type 2 or above bleeding $(\mathrm{RR}=1.16$; $95 \% \mathrm{Cl}=0.89-1.52 ; p=0.26, P^{2}=0 \%$ ), stent thrombosis ( $\mathrm{RR}=$ $1.58 ; 95 \% \mathrm{Cl}=0.90-2.76 ; p=0.11, R^{2}=0 \%$ ), and all-cause death $\left(\mathrm{RR}=1.10 ; 95 \% \mathrm{Cl}=0.86-1.43 ; p=0.45, P^{2}=0 \%\right)$ except $\mathrm{MI}\left(\mathrm{RR}=1.38 ; 95 \% \mathrm{Cl}=1.05-1.81 ; p=0.02, I^{2}=0 \%\right)$ Conclusion: Compared with prasugrel, ticagrelor did not reduce the primary composite endpoint of $\mathrm{Ml}$, stroke, and cardiovascular death at a weighted mean follow-up of 11 months. There was no significant difference between the secondary outcomes except MI.

(c) 2021 The Author(s).

Published by S. Karger AG, Basel

\section{Introduction}

$\mathrm{P} 2 \mathrm{Y}_{12}$ inhibitors are effective antiplatelet agents which act by blocking $\mathrm{P}_{2} \mathrm{Y}_{12}$ receptors on the platelet. $\mathrm{P}_{2} \mathrm{Y}_{12}$ inhibitors in combination with aspirin are recommended as dual antiplatelet therapy regimens in European guidelines
Correspondence to:

Ming-Yen Ng, myng2@hku.hk 

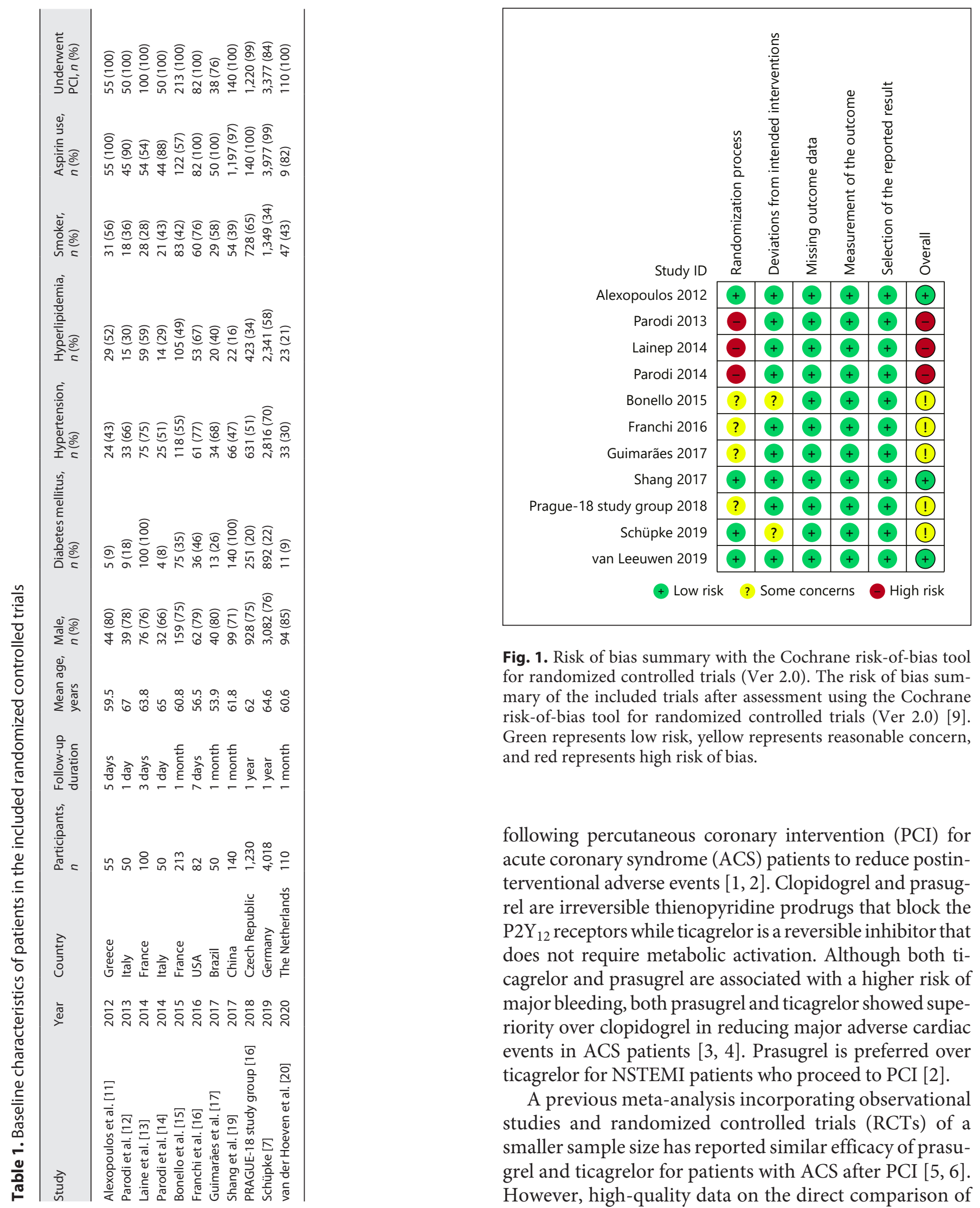

Fig. 1. Risk of bias summary with the Cochrane risk-of-bias tool for randomized controlled trials (Ver 2.0). The risk of bias summary of the included trials after assessment using the Cochrane risk-of-bias tool for randomized controlled trials (Ver 2.0) [9]. Green represents low risk, yellow represents reasonable concern, and red represents high risk of bias.

following percutaneous coronary intervention (PCI) for acute coronary syndrome (ACS) patients to reduce postinterventional adverse events $[1,2]$. Clopidogrel and prasugrel are irreversible thienopyridine prodrugs that block the $\mathrm{P} 2 \mathrm{Y}_{12}$ receptors while ticagrelor is a reversible inhibitor that does not require metabolic activation. Although both ticagrelor and prasugrel are associated with a higher risk of major bleeding, both prasugrel and ticagrelor showed superiority over clopidogrel in reducing major adverse cardiac events in ACS patients [3,4]. Prasugrel is preferred over ticagrelor for NSTEMI patients who proceed to PCI [2].

A previous meta-analysis incorporating observational studies and randomized controlled trials (RCTs) of a smaller sample size has reported similar efficacy of prasugrel and ticagrelor for patients with ACS after PCI $[5,6]$. However, high-quality data on the direct comparison of 
Fig. 2. Search and screening approach. The flow of trial reports identified and screened in this analysis. The search recovered 888 reports. Eight hundred fifty-five reports were screened after removing duplicates. Eleven reports were included after screening, and reasons for exclusion were stated.
Reports identified from search $(n=888)$

Duplicate reports $(n=33)$

Reports for screening $(n=855)$

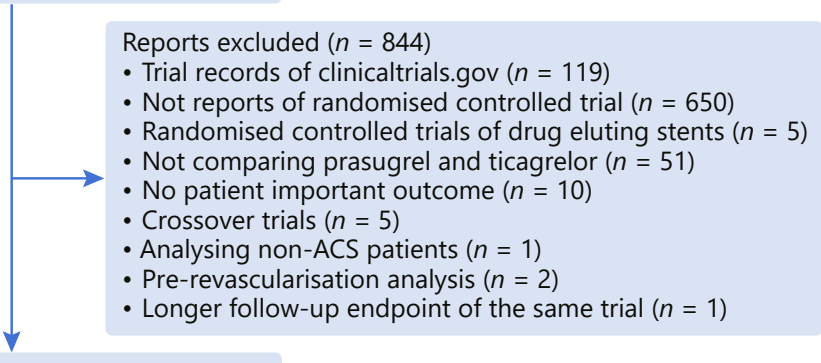

Reports included in the PT ACS study $(n=11)$ prasugrel and ticagrelor have been limited until the recent ISAR-REACT 5 trial. Prasugrel demonstrated superiority over ticagrelor in reducing major adverse cardiac events, which was defined as a composite endpoint of death, myocardial infarction, and stroke, with both of them sharing a similar risk of bleeding tendency [7]. In light of the discrepancies of previous studies, we conducted a meta-analysis of RCTs comparing the efficacy of prasugrel and ticagrelor in patients with ACS with respect to clinical outcomes by summarizing the evidence available.

\section{Methods}

TheprotocolwasregisteredonPROSPERO(CRD42020166810). This study was performed following the PRISMA guidelines [8].

\section{Eligibility Criteria}

Reports describing RCTs comparing the use of prasugrel and ticagrelor as loading dose and maintenance dose for patients with acute coronary artery disease up to February 2020 were included. Crossover trials, trials reported in research letters, and conference abstracts were excluded. In situations where there were multiple reports describing trial results with various follow-up endpoints, the report with the longest follow-up duration data was chosen over the others.

\section{Quality Assessment}

Risk of bias and quality assessment for the included trials was done using the Cochrane risk-of-bias tool version 2 (shown Fig. 1) [9]. Publication bias was assessed for all the outcome parameters with the funnel plot asymmetry using standard errors as measures of study size and risk ratios (RR) for treatment effect.

\section{Literature Search}

Reports of RCTs up to February 2020 were extracted from online databases MEDLINE, Embase, clinicaltrials.gov, and WHO's
International Clinical Trials Registry Platform using Cochrane Central Register of Controlled trials (CENTRAL) and OVID, respectively (online suppl. methods 1; for all online suppl. material, see www.karger.com/doi/10.1159/000520673) (L.C.W.F.). The combination of search terms "acute coronary syndrome," "randomized controlled trials," "coronary artery disease," "prasugrel," and "ticagrelor" was used to recover results. The search was limited to English and translated studies. Two independent reviewers (L.C.W.F. and N.H.C.L.) screened the search results by reviewing the abstracts and the titles against the inclusion and exclusion criteria. Reference lists of eligible articles were reviewed for further potential citations. Potentially eligible reports were identified, and manuscripts were reviewed thoroughly by 2 independent reviewers. Disagreements were resolved by consensus, with the assistance of a third reviewer where required (M.-Y.N.)

\section{Outcomes and Definitions}

The primary clinical endpoint was a composite of stroke, myocardial infarction, and cardiovascular death. Secondary outcomes including stroke, myocardial infarction, cardiovascular death, major bleeding defined as Bleeding Academic Research Consortium (BARC) type 2 or above bleeding [10], stent thrombosis, all-cause death, and other side effects were evaluated separately as individual outcomes. Baseline information of the trial population characteristics, study design, and outcome data regardless of being reported as the primary outcome, secondary outcome, or safety outcomes was extracted from the included reports. Data were extracted by one of the reviewers based on the agreed inclusion list of RCTs (L.C.W.F.). When study data were incomplete for analysis, investigators of the included trials were contacted for clarification.

\section{Statistical Analysis}

Review Manager (RevMan) (Version 5.3; Copenhagen: The Nordic Cochrane Centre, The Cochrane Collaboration, 2014) and SPSS (Released 2019. IBM SPSS Statistics for Windows, Version 26.0.; IBM Corp., Armonk, NY, USA) were used for the statistical analysis of the outcome data. Weighted mean follow-up duration was calculated with respect to the sample size of studies. RR with $95 \%$ confidence interval (CI) was estimated using the random-ef- 


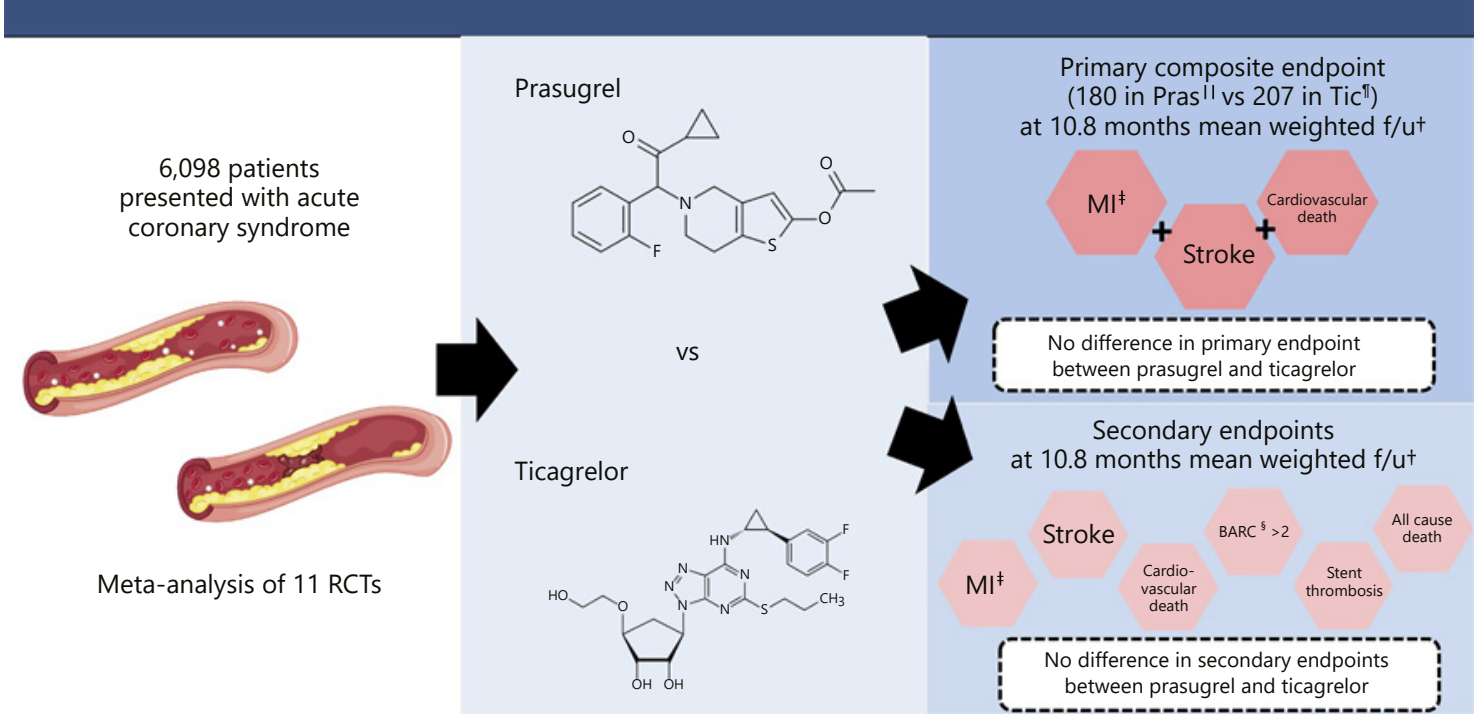

Fig. 3. Central illustration: comparing the efficacy of prasugrel and ticagrelor among acute coronary syndrome patients*. The graphical summary of the study. From the 11 eligible RCTs, we identified 6,098 patients with acute coronary syndrome who were randomized to either prasugrel or ticagrelor with a weighted mean followup of 11 months. There is no difference in the primary composite endpoint of myocardial infarction, stroke, and cardiovascular death between the use of prasugrel and ticagrelor after analyzing the outcome data using the random-effects model $(\mathrm{RR}=1.17 ; 95 \%$ $\left.\mathrm{CI}=0.96-1.42 ; p=0.12, I^{2}=0 \%\right)$. "Atherosclerosis image: Freepik.com designed by brfx." This cover has been designed using resources from Freepik.com; ${ }^{\dagger} \mathrm{f} / \mathrm{u}=$ follow-up; ${ }^{*} \mathrm{MI}$, myocardial infarction; ${ }^{\circledR}$ BARC, Bleeding Academic Research Consortium; "Pras, prasugrel; 'Tic, ticagrelor. fects method for analysis. A random-effect model was chosen for the analysis after considering the difference in ACS proportions (STEMI/NSTEMI), treatment strategies, and possible heterogeneity in the included RCTs. The estimate of effect was summarized by incorporating the clinical outcomes as RR with 95\% CI. Heterogeneity testing was performed using the Mantel-Haenszel method, and significant heterogenicity was shown by $I^{2}$ with a threshold of $>50 \%$. A prespecified sensitivity analysis on the primary composite outcome was performed by stratifying trials into long-term $(>1$ month) and short-term ( $=1$ month and $<1$ month) outcome data. The sensitivity analysis aimed to explore any significant variation of efficacy with time.

\section{Results}

After screening for eligibility and excluding duplicates, 11 studies were included from a total of 888 recovered from the initial search (shown in Fig. 2) [7, 11-20]. Baseline study characteristics of the included trials are reported in Table 1. Six thousand ninety-eight patients were included, with a mean age of $61 \pm 4$ years (24\% female). Three thousand fifty patients (50\%) were randomized to the prasugrel arm while 3,048 patients (50\%) were randomized to the ticagrelor arm with a weighted mean follow-up of $10.6 \pm 2.45$ months. In total, there were 180 primary composite endpoint events in the prasugrel arm and 207 primary composite endpoint events in the ticagrelor arm, respectively; 272 patients (4\%) were lost to follow-up. Overall, $76 \%$ were male, $50 \%$ with ST elevation MI, 35\% with non-ST elevation MI, 15\% with unstable angina, $25 \%$ with diabetes mellitus, $64 \%$ with hypertension, $51 \%$ with hyperlipidemia, and $42 \%$ smokers.

\section{Quality Assessment Results}

Risk of bias summary after assessment with the Cochrane risk-of-bias tool is summarized and shown in Figure 1. Three earlier trials were under high risk of bias due to poor integrity over the allocation concealment, while another 4 trials did not clearly report the method of ensuring allocation concealment. Two of the included trials 
Composite Endpoint of MI, Stroke and Cardiovascular Death

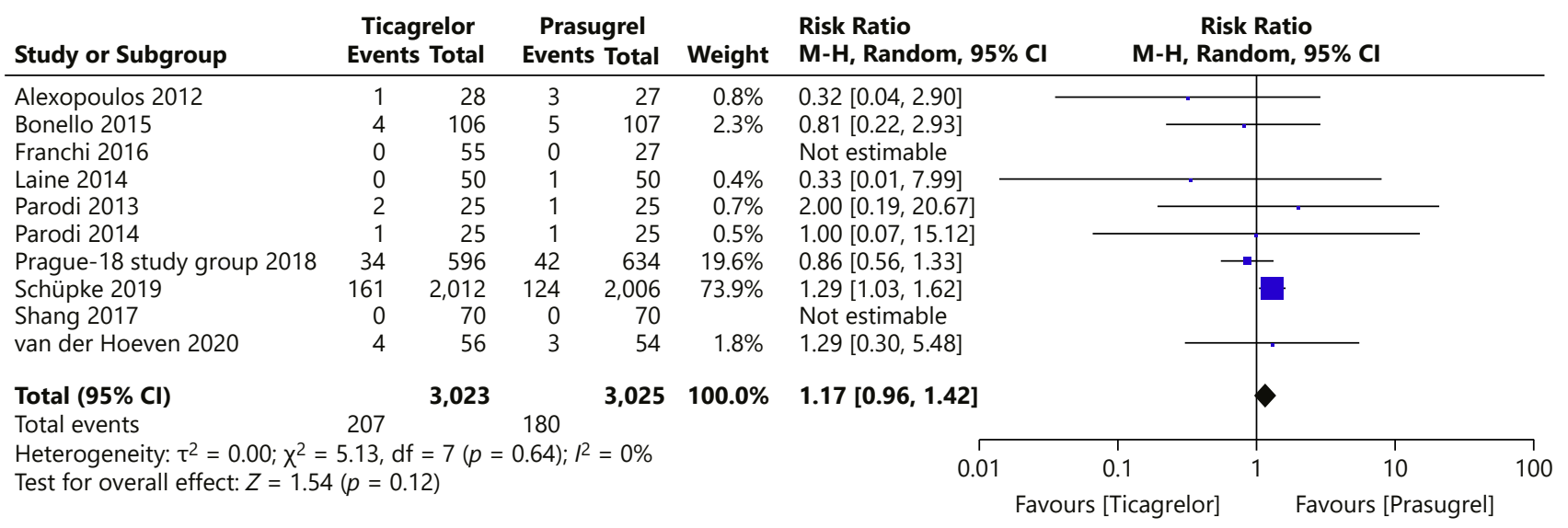

Fig. 4. Forest plot of the primary outcome: composite of stroke, myocardial infraction, and cardiovascular death according to the prasugrel or ticagrelor treatment group. The forest plot for the primary composite endpoint of stroke, myocardial infarction, and cardiovascular death from 10 of the 11 included RCTs. There is no statistical significance between the risk of using prasugrel or ticagrelor for patients with ACS on the primary composite endpoint $\left(\mathrm{RR}=1.17 ; 95 \% \mathrm{CI}=0.96-1.42 ; p=0.12, I^{2}=0 \%\right)$. M-H, Mantel-Haenszel.

were investigator-blinded trials while 6 were open-labeled trials. Three trials did not clearly state if blinding procedures were in place. Otherwise, risk of other bias was minimal.

\section{Primary Outcome}

The primary composite endpoint of stroke, myocardial infarction, and cardiovascular death was reported in 10 of the 11 eligible RCTs with only 6 of the 11 studies recording events (shown in Fig. 3). For the ISAR-REACT 5 trial, an appropriate endpoint outcome composing of death, myocardial infarction, and cardiovascular death was not reported in the published manuscript. From email correspondence with the investigators of ISAR-REACT 5, we obtained the appropriate composite endpoint for ticagrelor and prasugrel allowing a more precise estimate. ISARREACT 5 investigators reported 161 events in the ticagrelor group and 124 events in the prasugrel group. There were 122 cardiovascular death events (63 in the ticagrelor group and 59 in the prasugrel group). Compared to prasugrel, ticagrelor showed similar efficacy in reducing the risk of the primary composite endpoint (shown in Fig. 4) (RR $\left.=1.17 ; 95 \% \mathrm{CI}=0.96-1.42 ; p=0.12, I^{2}=0 \%\right)$. The funnel plot of the primary composite endpoint showed asymmetry with "left side corner effect" (shown in Fig. 5). Last, bias cannot be excluded based on the asymmetrical funnel plot, although Egger's test did not indicate this $(p=0.186)$.

Comparison of Prasugrel and Ticagrelor for ACS: The PT-ACS Study

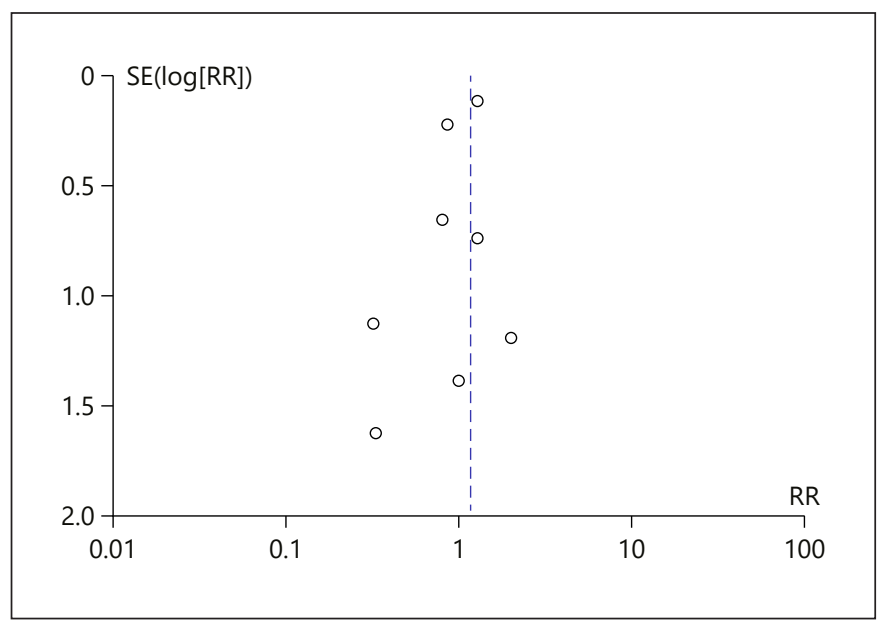

Fig. 5. Funnel plot of the primary composite outcome. The funnel plot for the primary composite outcome to assess the possibility of publication bias. The prasugrel group is used as the reference group in the RR pool estimate. Asymmetry was shown on the funnel plot with a "left side corner effect." SE, standard error; RR, risk ratio.

\section{Secondary Outcomes}

Eleven RCTs assessed the secondary outcomes to compare the efficacy of prasugrel and ticagrelor (Table 2), reporting a total of 54 stroke events, 206 myocardial infarction events, 169 cardiovascular death events, 


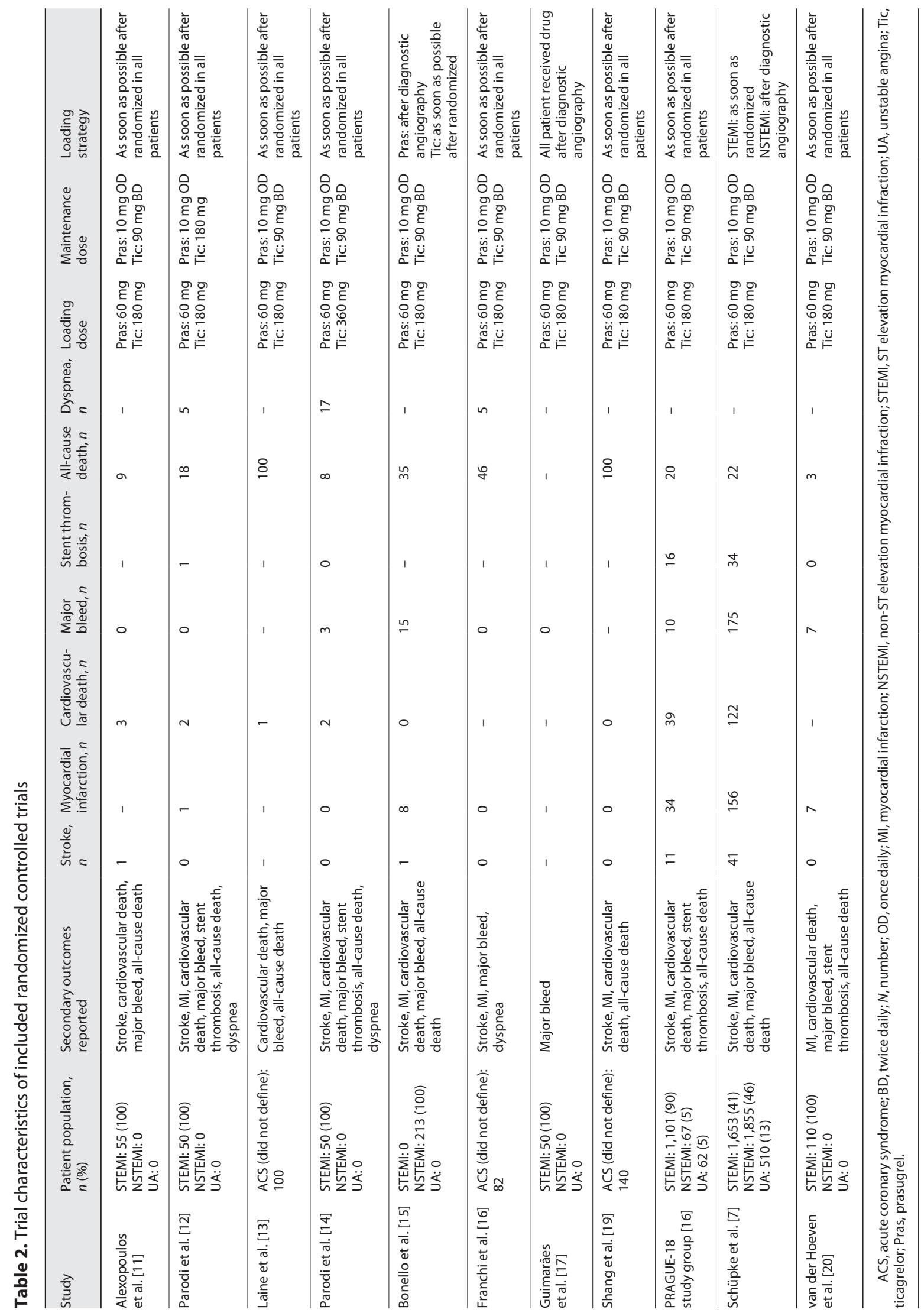




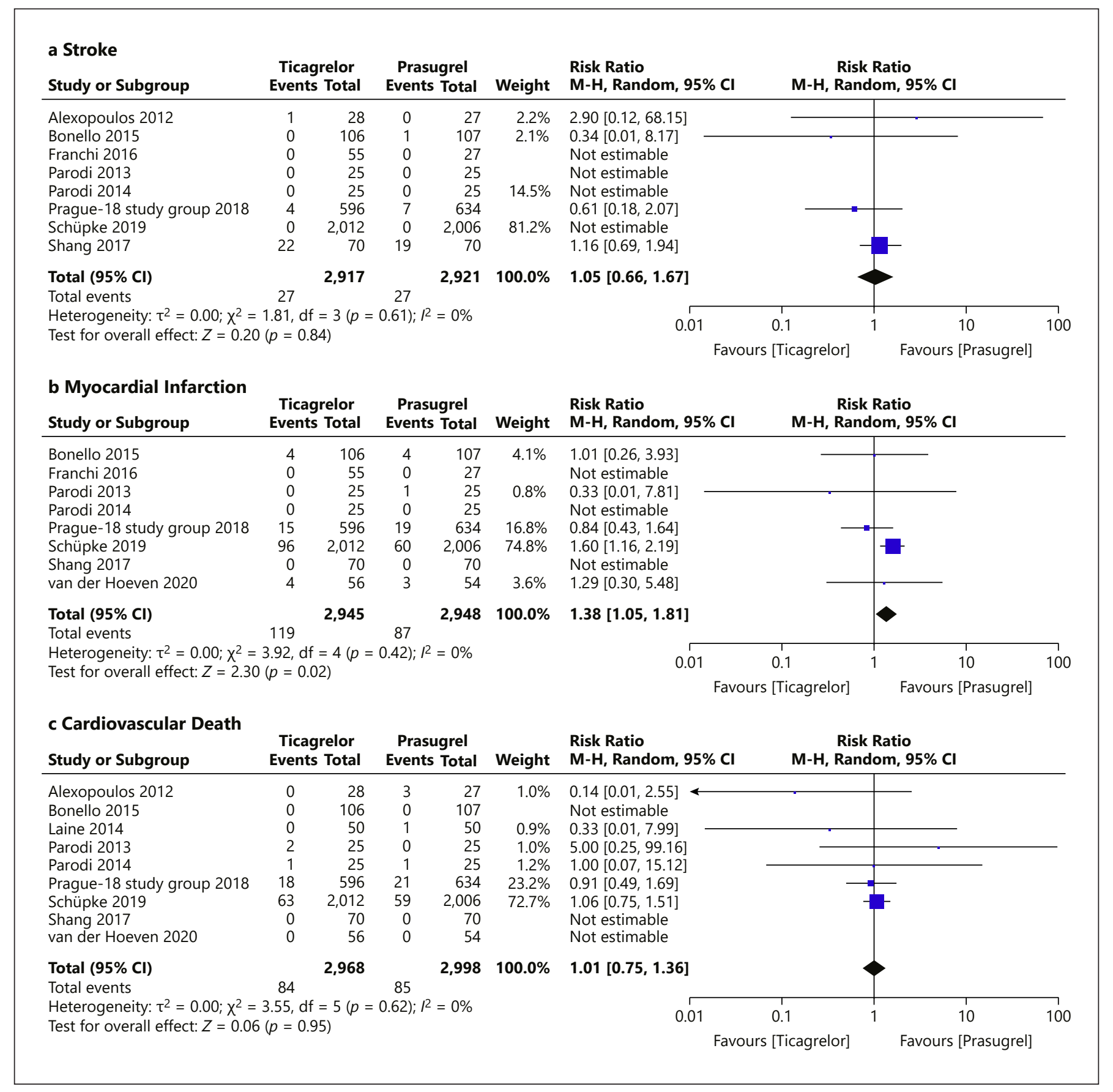

Fig. 6. Forest plot of secondary outcomes: according to prasugrel or ticagrelor treatment strategy. The effect estimates using a random-effects model of the secondary outcomes included stroke, myocardial infarction, cardiovascular death, major bleed, stent thrombosis, and all-cause death. There was no significant risk difference between using ticagrelor and prasugrel with respect to all secondary outcomes except myocardial infarction. $\mathrm{M}-\mathrm{H}$, Mantel-Haenszel.

(Figure continued on next page.) 


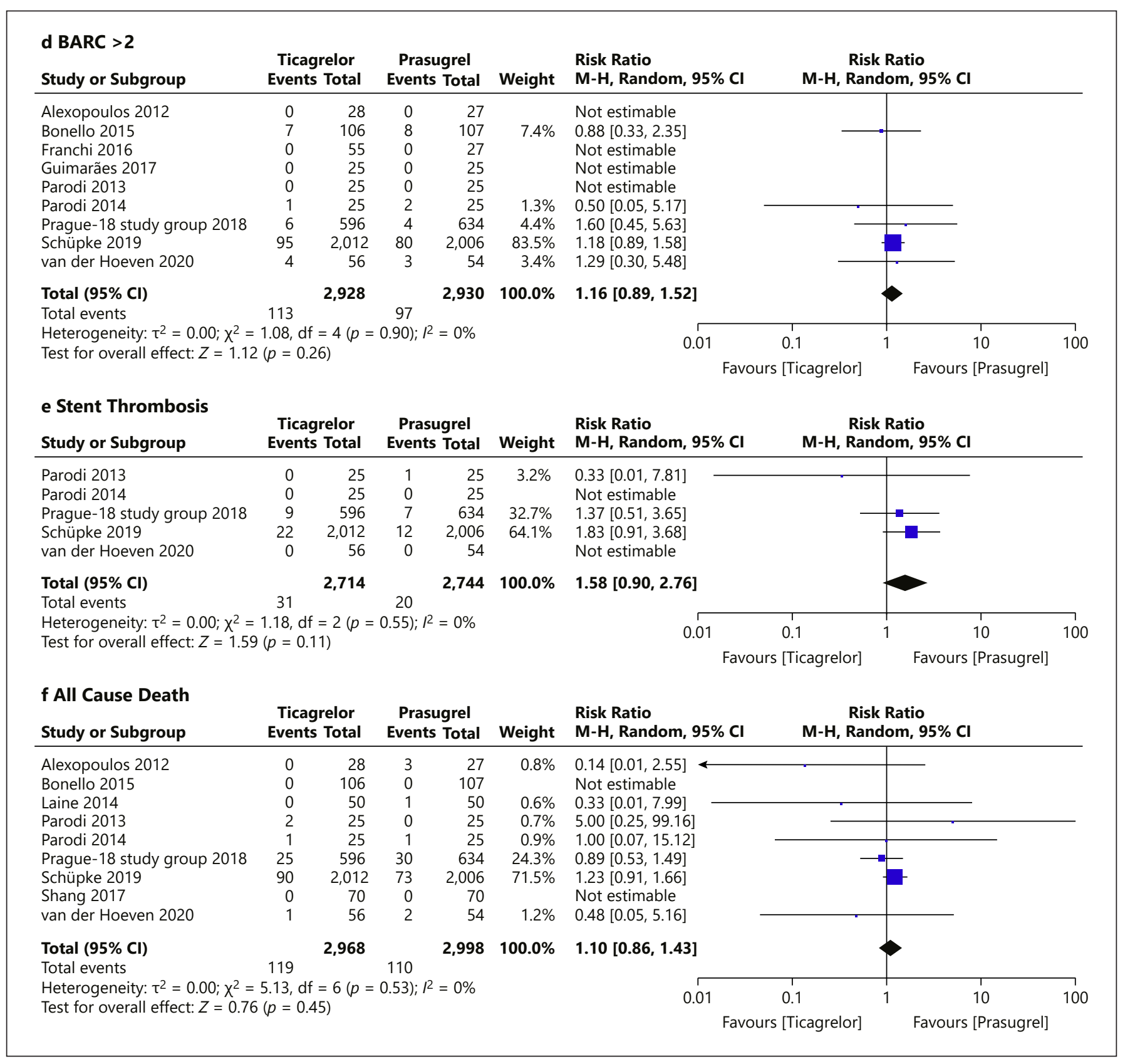

210 major bleeding events, 51 stent thrombosis events, and 229 all-cause death events. Prasugrel showed superiority over ticagrelor in reducing myocardial infarction events $\left(\mathrm{RR}=1.38 ; 95 \% \mathrm{CI}=1.05-1.81 ; p=0.02, I^{2}=0 \%\right)$. Otherwise, there was no significant difference between ticagrelor and prasugrel in reducing stroke $(\mathrm{RR}=1.05$; 95\% CI $\left.=0.66-1.67 ; p=0.84, I^{2}=0 \%\right)$, cardiovascular death $\left(\mathrm{RR}=1.01 ; 95 \% \mathrm{CI}=0.75-1.36 ; p=0.95, I^{2}=0 \%\right)$, BARC type 2 or above bleeding $(\mathrm{RR}=1.17 ; 95 \% \mathrm{CI}=$ $\left.0.90-1.54 ; p=0.24, I^{2}=0 \%\right)$, stent thrombosis $(\mathrm{RR}=1.58$; 95\% CI $\left.=0.90-2.76 ; p=0.11, I^{2}=0 \%\right)$, and all-cause death $\left(\mathrm{RR}=1.10 ; 95 \% \mathrm{CI}=0.86-1.43 ; p=0.45, I^{2}=0 \%\right)$ (shown in Fig. 6).

Dyspnea episodes were reported in 3 of the 11 included RCTs with a weighted mean follow-up of $3 \pm 2$ days as one of the side effects. Ticagrelor usage was associated with a significant increased risk of dyspnea (Fig. 7) $(\mathrm{RR}=$ $\left.7.70 ; 95 \% \mathrm{CI}=1.21-49.12 ; p=0.03, I^{2}=37 \%\right)$. 
Random effects: Secondary Endpoint: Dyspnoea

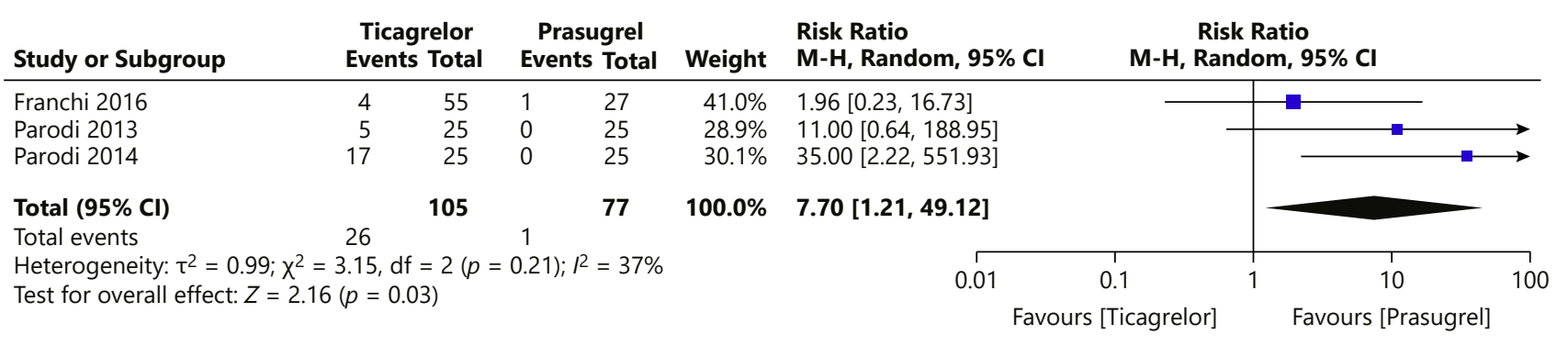

Fig. 7. Forest plot of dyspnea: according to prasugrel or ticagrelor treatment strategy. The effect estimates using a random-effects model of dyspnea were analysed. There was significant risk increase associated with the use of ticagrelor compared to prasugrel $\left(\mathrm{RR}=7.70 ; 95 \% \mathrm{CI}=1.21-49.12 ; p=0.03, I^{2}=37 \%\right)$. M-H, Mantel-Haenszel.

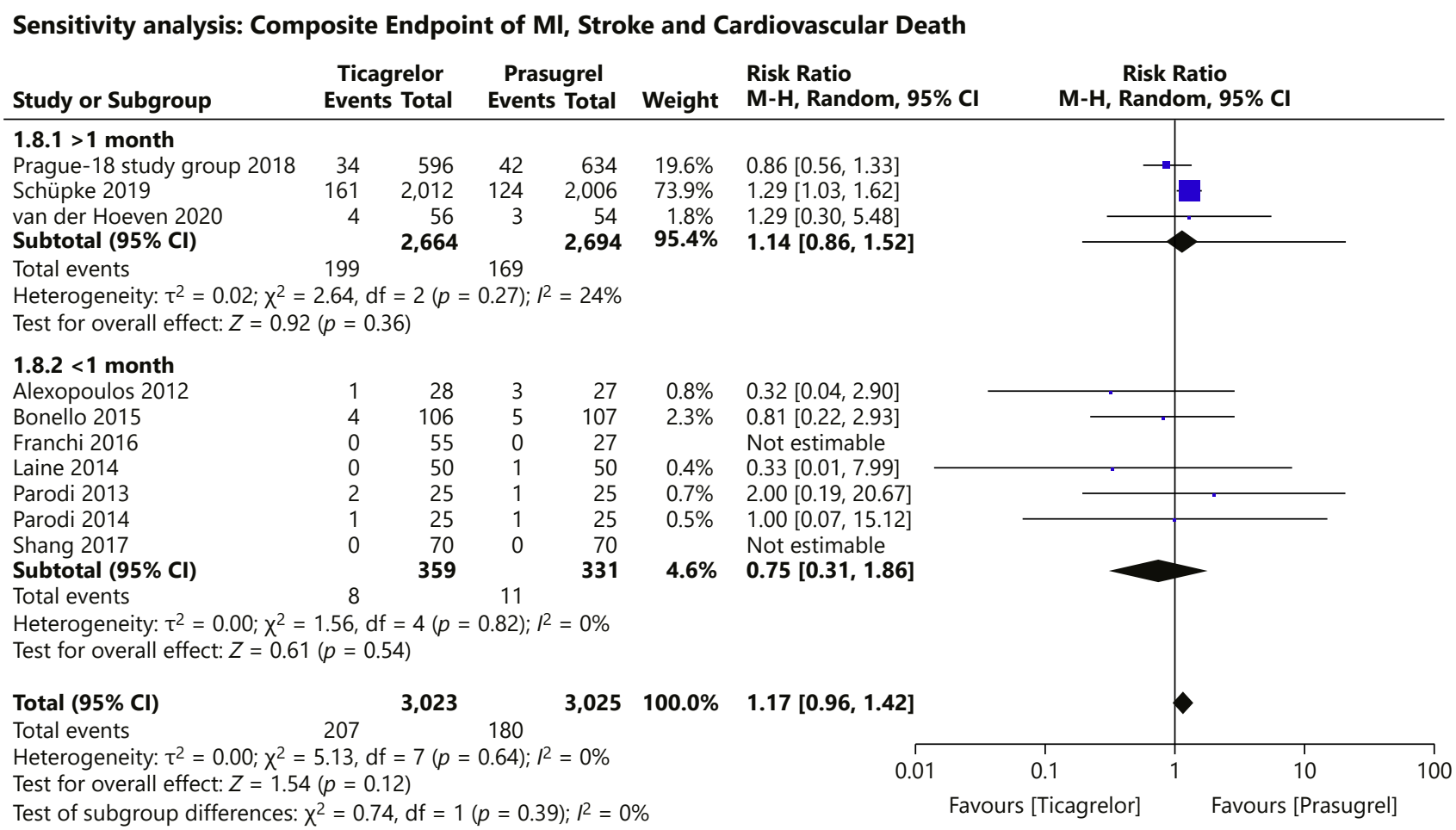

Fig. 8. Sensitivity analysis of the primary composite endpoint stratified by length of follow-up. A prespecified sensitivity analysis stratifying the trials by the duration of follow-up was assessed for the primary composite outcome. There was no risk difference between the use of prasugrel and ticagrelor among all of the 3 subgroups, respectively ( $>1$ month: $\mathrm{RR}=1.14 ; 95 \% \mathrm{CI}=0.86-1.52 ; p=0.36, I^{2}=24 \%$; $=1$ month: $\mathrm{RR}=0.81 ; 95 \% \mathrm{CI}=0.22-$ $2.93 ; p=0.74 ;<1$ month: $\left.\mathrm{RR}=0.71 ; 95 \% \mathrm{CI}=0.20-2.51 ; p=0.59, I^{2}=0 \%\right)$.

\section{Sensitivity Analysis}

A prespecified sensitivity analysis was carried out to explore any potential association between risk reduction with time of follow-up in the primary outcome. Three
RCTs followed up patients with ACS for $>1$ month, 2 RCTs had a follow-up duration of 1 month, and 5 RCTs followed up for $<1$ month (shown in Fig. 8). All subgroups did not show significant difference in risk between 
the allocated treatment groups $(>1$ month: $\mathrm{RR}=1.14$; 95\% CI $=0.86-1.52 ; p=0.36, I^{2}=24 \%$; =1 month: $\mathrm{RR}=$ $0.81 ; 95 \% \mathrm{CI}=0.22-2.93 ; p=0.74 ;<1$ month: $\mathrm{RR}=0.71$; $\left.95 \% \mathrm{CI}=0.20-2.51 ; p=0.59, I^{2}=0 \%\right)$.

\section{Discussion}

The current review of RCTs comparing prasugrel and ticagrelor in ACS showed no significant risk difference in the primary composite end point of stroke, myocardial infarction, and cardiovascular death with a weighted mean follow-up of 11 months. There is no difference in risk between all of the secondary outcomes in ACS except in myocardial infarction.

\section{Prasugrel versus Ticagrelor}

Clinical data on comparing the efficacy of prasugrel and ticagrelor have been limited before the release of the ISAR-REACT 5 trial result. There were a number of small RCTs comparing the pharmacological activity of the agents, but their analyses were not on clinical events [21, 22 ]. Small RCTs that reported and compared clinical outcomes were of short follow-up duration ( $<1$ month) and small sample size (under 100) [11-14]. Most data before ISAR-REACT 5 indicated a similar efficacy between prasugrel and ticagrelor [23]. Clinicians often refer to the 2 landmark RCTs, PLATO and TRITON-TIMI, comparing prasugrel and ticagrelor to clopidogrel, respectively, for an indirect comparison which showed that prasugrel was associated with a higher risk of major bleeding. Given that ticagrelor and prasugrel are both superior over clopidogrel, this indicated a potential that ticagrelor should be considered over prasugrel after balancing the bleeding risk $[3,4]$.

The PRAGUE-18 trial was the first large RCT directly comparing prasugrel and ticagrelor in the long term, which found no difference between the two in safety and efficacy. However, the study was subject to methodological issues including a high switching rate to clopidogrel, premature termination, and statistical underpower, which limited the interpretation of PRAGUE-18 trial results. The PRAGUE-18 trial population is STEMI predominant (89\%) which affects the generalizability of the data to NSTEMI patients and potentially misrepresents the effect among NSTEMI patients. Therefore, ISAR-REACT 5 was designed and conducted. ISAR-REACT 5 showed that prasugrel was superior to ticagrelor in reducing the primary composite endpoint $(\mathrm{RR}=1.29 ; 95 \% \mathrm{CI}$ $=1.03-1.62$ ) [7]. However, ISAR-REACT 5 is an open- label trial. There is also difference in study drug discontinuation rates among the ticagrelor and prasugrel group $(15.2 \%$ vs. $12.5 \%$, respectively, $p=0.03)$ and a significantly longer duration of treatment before discontinuation in the prasugrel group (median: 84 days in the ticagrelor group vs. median: 109 days in the prasugrel group, $p=$ $0.01)$. These factors might have partly accounted for the difference in primary outcome incidence $(10 \%$ in the ticagrelor group vs. 9.3\% in the prasugrel group) resulting in overestimation of prasugrel benefit. ISAR-REACT 5 and PRAGUE-18 are both European-based studies which limit the generalizability of the data. Therefore, this metaanalysis was conducted to review all available evidence on comparing ticagrelor and prasugrel to further explore the uncertainty and inconsistency of the different studies. We hypothesized that a meta-analysis would show that prasugrel was superior by including the ISAR-REACT 5 trial. By including ISAR-REACT 5 to studies from Asia, Europe, North America, and South Africa, this meta-analysis' results would be more generalizable for clinicians worldwide.

While ISAR-REACT 5 data contributed over $70 \%$ of the summary estimate, prasugrel is not superior to ticagrelor for ACS patients in reducing the primary composite outcome risk $(\mathrm{RR}=1.17 ; 95 \% \mathrm{CI}=0.96-1.42 ; p=$ $\left.0.12, I^{2}=0 \%\right)$. This meta-analysis highlights the uncertainty of the prasugrel superiority in terms of the composite outcome. However, from the 95\% CI, ticagrelor is limited to a relative risk decrease by $4 \%$ at best, while potentially worse than prasugrel by a relative risk increase of $42 \%$. This implies that although ticagrelor can be an alternative to prasugrel as a more potent antiplatelet agent than clopidogrel, clinicians should consider the potentially lower efficacy of ticagrelor compared with prasugrel.

The result was in line with previous meta-analyses. Multiple previous meta-analyses comparing the use of prasugrel and ticagrelor demonstrated no difference in risk among both ACS patients and type 2 diabetic mellitus patients $[5,6,23,24]$. Due to limited data, results from observational studies had to be included in the pool estimate of previous meta-analysis, and the mean follow-up duration was 1 month [5]. A recent meta-analysis included ISAR-REACT 5 results [25]. However, investigators included the PRAGUE-18 population twice in the analysis which might have biased the result. Therefore, this meta-analysis which included worldwide RCT data only provided a more comprehensive comparison after incorporating the recent large RCT results [7, 19]. A recent network meta-analysis comparing prasugrel, ticagrelor,
Fong/Lee/Yan/Ng 
and clopidogrel had similar finding to this meta-analysis finding. The investigators found no difference in the efficacy and safety difference between prasugrel and ticagrelor by combining results from PRAGUE-18 and ISAR-REACT 5 [26].

Prasugrel was only superior to ticagrelor in the secondary myocardial infarction outcome with low heterogeneity $\left(\mathrm{RR}=1.38 ; 95 \% \mathrm{CI}=1.05-1.81 ; p=0.02, I^{2}=0 \%\right)$. Although prasugrel was superior to ticagrelor in the MI outcome, better stratification in the definition of MI by fatal and nonfatal might yield alternative result. Since there were 2 trials that did not clearly state if it was fatal or nonfatal MI, our methodology would be enhanced if there were more transparency about the data extracted $[12,15]$. None of the other secondary outcomes included in this meta-analysis showed significant risk differences between the use of prasugrel and ticagrelor estimated by the random-effects model (shown in Fig. 6). Three studies among the 11 included studies noted increased risk of dyspnea associated with ticagrelor use. This reiterates dyspnea as one of the acute side effects of ticagrelor which is reported and recognized in previous secondary analysis and cohort studies $[27,28]$. However, the chronicity of dyspnea associated with ticagrelor use cannot be established in this analysis due to the limited long-term data.

In the prespecified sensitivity analysis that stratified trials by follow-up duration, no significant result was yielded in the long-term outcome group. However, high heterogeneity was also reported $\left(I^{2}=71 \%\right)$. It was due to the limited number of studies $(n=3)$ that investigated prasugrel and ticagrelor in long term. The current evidence showed a contradictory result from the ISAR-REACT 5 and PRAGUE-18, which are the current 2 largest RCTs providing head to head comparison of the agents $[7,19]$. The conflicting result could be explained by the premature termination, underpower, and slight difference in patient population build-up in the PRAGUE-18 trial. This highlights the need for more data before drawing an accurate conclusion on the comparison between prasugrel and ticagrelor [29].

\section{Limitations}

Our study had several limitations. First, over half of the trials had issues with the integrity of allocation concealment. The reports did not clearly state the way concealment was maintained, which by standard should involve the use of sequentially numbered, sealed, opaque envelopes or central randomization with a computer [30]. This might have introduced bias. On the outcome level, long-term efficacy of the antiplatelet agents could not be compared due to the lack of long-term data ( $>1$ year). In the prespecified time-stratified analysis, there is substantial heterogeneity noted in the longer follow-up group $(>1$ month) (shown in Fig. 8). Second, the funnel plots (shown in Fig. 5) showed potential asymmetry, but Egger's test was insignificant. The accuracy of the summary estimate may be compromised as selective reporting might have created bias in the present data. Therefore, the effect of prasugrel could have been underestimated. Last, the number of events for the secondary outcomes is limited by the small number of studies included. This has limited our ability to draw a concrete conclusion on the comparison of ticagrelor and prasugrel. Stratified data by patient groups (STEMI vs. NSTEMI patients) are not provided by the included studies, limiting our ability to further explore the difference in ticagrelor and prasugrel efficacy among STEMI and NSTEMI patients.

\section{Future Directions}

Based on the current evidence, prasugrel and ticagrelor have similar efficacy. Therefore, the current guideline suggesting the use of either prasugrel or ticagrelor for ACS patients over clopidogrel is appropriate $[1,31]$. Future studies can investigate the long-term efficacy of prasugrel and ticagrelor. Additional information on their risk difference can shed light on the long-term prognosis helping clinicians understand the efficacy and risks of ticagrelor and prasugrel. Diabetes can be a significant confounder and therefore should be adjusted in future studies comparing the 2 agents $[32,33]$. The patents of the 2 agents are expiring. Although incentive for another comparison between the agents could be lowered, future comparisons of the agents are less susceptible to conflict of interest from industries sponsorship. Therefore, we hope this study will be able to assemble all current evidence that compared ticagrelor and prasugrel and provide a general picture for clinicians.

\section{Conclusions}

Compared with prasugrel, ticagrelor showed similar efficacy in reducing risk of the primary composite endpoint of MI, stroke, and cardiovascular death at a weighted mean follow-up of 11 months based on our findings. There was no significant difference between the secondary outcomes except myocardial infarction. 


\section{Statement of Ethics}

An ethics statement was not required for this study type.

\section{Conflict of Interest Statement}

L.C.W.F., N.H.C.L., and M.-Y.N. have no disclosures to make. A.T.Y. has received research grant support from Astra-Zeneca.

\section{Funding Sources} study.

The authors did not receive any funding for conducting the

\section{Author Contributions}

Lucas Chun Wah Fong contributed to conceptualization, methodology, software, formal analysis, and writing - original draft. Nicholas Ho Cheung Lee contributed to validation and writing - review and editing. Ming-yen Ng contributed to methodology, writing - review and editing, and supervision. Andrew Yan contributed to writing - review and editing and supervision.

\section{Data Availability Statement}

All data generated or analyzed during this study are included in this article and online supplementary material. Further enquiries can be directed to the corresponding authors.

\section{References}

1 Ibanez B, James S, Agewall S, Antunes MJ, Bucciarelli-Ducci C, Bueno H, et al. 2017 ESC Guidelines for the management of acute myocardial infarction in patients presenting with ST-segment elevation: the Task Force for the management of acute myocardial infarction in patients presenting with ST-segment elevation of the European Society of Cardiology (ESC). Eur Heart J. 2018 Jan 7;39(2):119-77.

2 Collet JP, Thiele $\mathrm{H}$, Barbato E, Barthélémy O, Bauersachs J, Bhatt DL, et al. 2020 ESC Guidelines for the management of acute coronary syndromes in patients presenting without persistent ST-segment elevation. Eur Heart J. 2021;42(14):1289-367.

3 Wiviott SD, Braunwald E, McCabe CH, Montalescot G, Ruzyllo W, Gottlieb S, et al. Prasugrel versus clopidogrel in patients with acute coronary syndromes. N Engl J Med. $2007 \mathrm{Nov}$ 15;357(20):2001-15.

4 Wallentin L, Becker RC, Budaj A, Cannon CP, Emanuelsson $\mathrm{H}$, Held C, et al. Ticagrelor versus clopidogrel in patients with acute coronary syndromes. N Engl J Med. 2009 Sep 10; 361(11):1045-57.

5 Watti H, Dahal K, Zabher HG, Katikaneni P, Modi K, Abdulbaki A. Comparison of prasugrel and ticagrelor in patients with acute coronary syndrome undergoing percutaneous coronary intervention: a meta-analysis of randomized and non-randomized studies. Int J Cardiol. 2017 Dec 15;249:66-72.

6 Yang H, Tang B, Xu CH, Ahmed A. Ticagrelor versus prasugrel for the treatment of patients with type 2 diabetes mellitus following percutaneous coronary intervention: a systematic review and meta-analysis. Diabetes Ther. 2019 Feb;10(1):81-93.

7 Schüpke S, Neumann FJ, Menichelli M, Mayer K, Bernlochner I, Wohrle J, et al. Ticagrelor or prasugrel in patients with acute coronary syndromes. N Engl J Med. 2019 Oct 17; 381(16):1524-34.
8 Moher D, Shamseer L, Clarke M, Ghersi D, Liberati A, Petticrew M, et al. Preferred reporting items for systematic review and metaanalysis protocols (PRISMA-P) 2015 statement. Syst Rev. 2015 Jan 1;4:1

9 Sterne JAC, Savovic J, Page MJ, Elbers RG, Blencowe NS, Boutron I, et al. RoB 2: a revised tool for assessing risk of bias in randomised trials. BMJ. 2019 Aug 28;366:14898.

10 Mehran R, Rao SV, Bhatt DL, Gibson CM, Caixeta A, Eikelboom J, et al. Standardized bleeding definitions for cardiovascular clinical trials: a consensus report from the Bleeding Academic Research Consortium. Circulation. 2011 Jun 14;123(23):2736-47.

11 Alexopoulos D, Xanthopoulou I, Gkizas V, Kassimis G, Theodoropoulos KC, Makris G, et al. Randomized assessment of ticagrelor versus prasugrel antiplatelet effects in patients with ST-segment-elevation myocardial infarction. Circ Cardiovasc Interv. 2012;5(6): 797-804.

12 Parodi G, Valenti R, Bellandi B, Migliorini A, Marcucci R, Comito V, et al. Comparison of prasugrel and ticagrelor loading doses in STsegment elevation myocardial infarction patients: RAPID (Rapid Activity of Platelet Inhibitor Drugs) primary PCI study. J Am Coll Cardiol. 2013;61(15):1601-6.

13 Laine M, Frère C, Toesca R, Berbis J, Barnay $\mathrm{P}$, Pansieri $\mathrm{M}$, et al. Ticagrelor versus prasugrel in diabetic patients with an acute coronary syndrome. A pharmacodynamic randomised study. Thromb Haemost. 2014;111(2):273-8.

14 Parodi G, Bellandi B, Valenti R, Migliorini A, Marcucci R, Carrabba N, et al. Comparison of double $(360 \mathrm{mg})$ ticagrelor loading dose with standard $(60 \mathrm{mg})$ prasugrel loading dose in ST-elevation myocardial infarction patients: the Rapid Activity of Platelet Inhibitor Drugs (RAPID) primary PCI 2 study. Am Heart J. 2014;167(6):909-14.
15 Bonello L, Laine M, Cluzel M, Frere C, Mancini J, Hasan A, et al. Comparison of ticagrelor versus prasugrel to prevent periprocedural myonecrosis in acute coronary syndromes. Am J Cardiol. 2015;116(3):339-43.

16 Franchi F, Faz GT, Rollini F, Park Y, Cho JR, Thano E, et al. Pharmacodynamic Effects of Switching From Prasugrel to Ticagrelor: results of the Prospective, Randomized SWAP-3 Study. JACC Cardiovasc Interv. 2016;9(11):1089-98.

17 Guimaraes L, Genereux P, Silveira D, Pesaro AE, Falcao F, Barbosa BRC, et al. P2Y12 receptor inhibition with prasugrel and ticagrelor in STEMI patients after fibrinolytic therapy: analysis from the SAMPA randomized trial. Int J Cardiol. 2017;230:204-8.

18 Shang LL, Guo DD, Zhao HY, Quan AJ, Cao PG. Comparison of pharmacodynamic effects of ticagrelor vs prasugrel in type 2 diabetes mellitus patients with coronary heart disease. J Clin Pharm Ther. 2018;43(3):342-7.

19 Motovska Z, Hlinomaz O, Kala P, Hromadka M, Knot J, Varvarovsky I, et al. 1-Year Outcomes of Patients Undergoing Primary Angioplasty for Myocardial Infarction Treated With Prasugrel Versus Ticagrelor. J Am Coll Cardiol. 2018 Jan 30;71(4):371-81.

20 van der Hoeven NW, Janssens GN, Everaars $\mathrm{H}$, Nap A, Lemkes JS, de Waard GA, et al. Platelet inhibition, endothelial function, and clinical outcome in patients presenting with st-segment-elevation myocardial infarction randomized to ticagrelor versus prasugrel maintenance therapy: long-term follow-up of the REDUCE-MVI Trial. J Am Heart Assoc. 2020 Mar 3;9(5):e014411.

21 Alexopoulos D, Galati A, Xanthopoulou I, Mavronasiou E, Kassimis G, Theodoropoulos $\mathrm{KC}$, et al. Ticagrelor versus prasugrel in acute coronary syndrome patients with high onclopidogrel platelet reactivity following percutaneous coronary intervention: a pharmacodynamic study. J Am Coll Cardiol. 2012 Jul 17;60(3):193-9. 
22 Rollini F, Franchi F, Cho JR, DeGroat C, Bhatti M, Muniz-Lozano A, et al. A head-to-head pharmacodynamic comparison of prasugrel vs. ticagrelor after switching from clopidogrel in patients with coronary artery disease: results of a prospective randomized study. Eur Heart J. 2016 Sep 14;37(35):2722-30.

23 Bundhun PK, Shi JX, Huang F. Head to head comparison of Prasugrel versus Ticagrelor in patients with acute coronary syndrome: a systematic review and meta-analysis of randomized trials. BMC Pharmacol Toxicol. 2017 Dec 12;18(1):80

24 Khan MS, Memon MM, Usman MS, Alnaimat S, Khan SU, Khan AR, et al. Prasugrel vs. ticagrelor for acute coronary syndrome patients undergoing percutaneous coronary intervention: a systematic review and metaanalysis. Am J Cardiovasc Drugs. 2019 Oct: 19(5):465-76

25 Ray A, Najmi A, Khandelwal G, Jhaj R, Sadasivam B. Prasugrel versus ticagrelor in patients with acute coronary syndrome undergoing percutaneous coronary intervention: a systematic review and meta-analysis of randomized trials. Cardiovasc Drugs Ther. 2021; 35(3):561-74
26 Navarese EP, Khan SU, Kołodziejczak M, Kubica J, Buccheri S, Cannon CP, et al. Comparative efficacy and safety of oral P2Y(12) inhibitors in acute coronary syndrome: network meta-analysis of 52816 patients from 12 randomized trials. Circulation. 2020 Jul 14; 142(2):150-60.

27 Bonaca MP, Bhatt DL, Oude Ophuis T, Steg PG, Storey R, Cohen M, et al. Long-term tolerability of ticagrelor for the secondary prevention of major adverse cardiovascular events: a Secondary Analysis of the PEGASUS-TIMI 54 Trial. JAMA Cardiol. 2016 Jul 1;1(4):425-32.

28 Lombardi N, Lucenteforte E, Torrini M, Balzi D, Barchielli A, Mugelli A, et al. Ticagrelorrelated late-onset dyspnea as cause of emergency department visit: a 3-year outpatient study. J Cardiovasc Med. 2018 Jun;19(6):2849.

29 Motovska Z, Hlinomaz O, Miklik R, Hromadka M, Varvarovsky I, Dusek J, et al. Prasugrel versus ticagrelor in patients with acute myocardial infarction treated with primary percutaneous coronary intervention: multicenter randomized PRAGUE-18 Study. Circulation. 2016 Nov 22;134(21):1603-12.
30 Juni P, Altman DG, Egger M. Systematic reviews in health care: assessing the quality of controlled clinical trials. BMJ. $2001 \mathrm{Jul} 7$; 323(7303):42-6.

31 Roffi M, Patrono C, Collet JP, Mueller C, Valgimigli M, Andreotti F, et al. 2015 ESC guidelines for the management of acute coronary syndromes in patients presenting without persistent ST-segment elevation: task force for the management of acute coronary syndromes in patients presenting without persistent ST-segment elevation of the European Society of Cardiology (ESC). Eur Heart J. 2016 Jan 14;37(3):267-315.

32 Bittl JA, Yong CM, Sharma G. When to believe unexpected results for ticagrelor or prasugrel. JACC Cardiovasc Interv. 2020;13(19): 2248-50.

33 Ndrepepa G, Kastrati A, Menichelli M, Neumann FJ, Wöhrle J, Bernlochner I, et al. Ticagrelor or prasugrel in patients with acute coronary syndromes and diabetes mellitus. JACC Cardiovasc Interv. 2020 Oct 12;13(19): 2238-47. 